\title{
Automated learning multi-criteria classifiers for FLIR ship imagery classification
}

\author{
Khaled Jabeur \\ Defence R\&D Canada Valcartier \\ 2459, Pie-XI Blvd North, Val-Bélair \\ Quebec, G3J 1X5 Canada \\ khaled.jabeur@drdc-rddc.gc.ca
}

\author{
Adel Guitouni \\ Defence R\&D Canada Valcartier \\ 2459, Pie-XI Blvd North, Val-Bélair \\ Quebec, G3J 1X5 Canada \\ adel.guitouni@drdc-rddc.gc.ca
}

\begin{abstract}
This paper proposes an Automated Learning Method (ALM) based on Real-Coded Genetic Algorithm (RCGA) to infer the Multi-Criteria Classifiers (MCC) parameters. The Multi-Criteria Classifiers (or Multi-Criteria Classification Methods) considered are based on concordance and discordance concepts. A military database of 2545 Forward Looking Infra-Red (FLIR) images representing eight different classes of ships is therefore used to test the performance of these classifiers. The empirical results of MCC are compared with those obtained by other classifiers (e.g. Bayes and Dempster-Shafer classifiers). In this paper, we show the benefits of cross-fertilization of multi-criteria classifiers and information fusion algorithms.
\end{abstract}

Keywords: multi-criteria classifiers, concordance, discordance, military application, genetic algorithm, automated learning, similarity index.

\section{Introduction}

In the context of Multi-Criteria Decision Analysis (MCDA), many methods are proposed to assign objects to predefined categories or classes. These methods are known as Multi-Criteria Classifiers MCC or multicriteria sorting methods, depending on whether the categories are defined in nominal or ordinal way. In this paper, we focus on nominal MCC, particularly those based on concordance/discordance concepts [23]; these MCC are called Nominal Concordance/Discordancebased MCC (or NCD-based MCC). The major limitation of NCD-based MCC is the large number of parameters (e.g. discrimination thresholds, weights, reference alternatives, etc.) required to compute the membership degree for each object to a pre-defined category. In decision-aid context, these parameters are generally elicited using interactive approaches with the decisionmaker; to articulate his relational preference system. However, when the number of these parameters is large it will be difficult for the decision-maker to provide such information in a coherent way. In this case, an Automatic or Machine Learning Approach (ALM or MLA) might be interesting to inferring the information. This paper proposes a particular ALM -based on a Real-Coded Genetic Algorithm (RCGA)- to estimate the parameters of NCD-based MCC. We apply the proposed ALM in the context of image classification. A database of 2545 Forward Looking Infra-Red (FLIR) images representing eight different classes of military ships is used for empirical validation. Even if the purpose of the comparison might be seen limited, we present experimental results by comparing NCD-based MCC to other classifiers such as Bayes and Dempster-Shafer classifiers.

In this paper is based on our other paper accepted in this conference Fusion 2007 [16].This paper is organized as follows. Section 2 presents the main idea of NCD-based MCC and a mathematical model which optimizes their parameters in the context of an ALM. Section 3 proposes a RCGA to infer the parameters of the NCD-based MCC. Section 4 presents a brief description of the database used to test the performance of the different classifiers. In section 5, computational results of some NCD-based MCC are presented and compared with those obtained by other classifiers. Finally, conclusions, discussions and feature works are presented in section 6.

\section{NCD-based MCC: a brief review}

The concordance and discordance concepts were introduced by Roy [23] when he defined the outranking relation in ELECTRE I method. This relation might be defined as follows ([24], [27], [6]): an alternative $x_{i}$ outranks an alternative $x_{k}$ if and only if there are enough arguments to decide that $x_{i}$ is at least as good as $x_{k}$ (concordance concept) while there is no essential reason to refute that statement (discordance concept). Recently, Perny [22], Belacel [3] and Henriet [12] extended the above definition by developing an indifference relation measuring the similarity between two alternatives $x_{i}$ and $x_{k}$. The main idea of this relation is presented in Figure 1. It's on the basis of this relation that NCD-based MCC assign an alternative (project, object, image, etc.) to a predefined category. To our knowledge, there exist in the literature four NCD-based MCC: (i) TRI-NOMFC classifier [17], (ii) PROAFTN classifier [3], (iii) PIP and K-PIP classifiers [12] and (iv) FBI classifier [22]. The aim of these NCD-based MCC is to compute for each object $a_{i}$ a fuzzy number called $\mu\left(a_{i}, C^{h}\right) \in[0,1]$, 
which measures its the membership degree to a given category $C^{h}$. Based on these membership degrees, an object $a_{i}$ is assigned to the category which has "the most common features" with $a_{i}$.

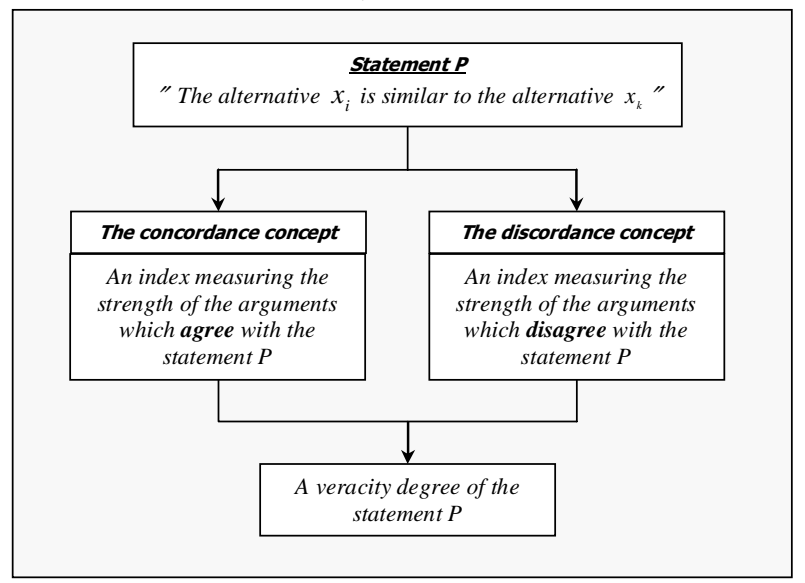

Figure 1. The main idea of the indifference relation.

The NCD-based MCC proceeds in five steps to assign an object $a_{i}$ to a predefined category $C^{h}$ :

1. For each criterion $g_{j}(j=1 . . n)$ and for each profile $b_{k}^{h}\left(k=1 . . L_{h}\right)$ characterizing $C^{h 1}(h=1 . . H)$, a local concordance $C_{j}\left(a_{i}, b_{k}^{h}\right)$ and discordance $D_{j}\left(a_{i}, b_{k}^{h}\right)$ indices are computed for each pair of alternatives $\left(a_{i}, b_{k}^{h}\right)$. The computation of these local indices takes into account the following three types of thresholds: indifference, preference and veto thresholds.

2. The concordance (respectively discordance) local indices are then aggregated into global indices $C\left(a_{i}, b_{k}^{h}\right) \quad$ (respectively $\left.D\left(a_{i}, b_{k}^{h}\right)\right)$ by using, in general, the weighted sum (respectively weighted geometric mean).

3. Global concordance and discordance indices are aggregated to compute the similarity degree $S I$ between the object $a_{i}$ and the profile $b_{k}^{h}$. In general, two kinds of aggregation operator are used to combine the quantities $C\left(a_{i}, b_{k}^{h}\right)$ and $\left(1-D\left(a_{i}, b_{k}^{h}\right)\right)$ : the Product and the Minimum.

4. Based on these similarity indices, an aggregation operator (usually the Maximum operator) is used to compute computes the membership degree $\mu\left(a_{i}, C^{h}\right)$ of $a_{i}$ to $C^{h}$.

5. Finally, the object $a_{i}$ will be assigned to the category $C^{h}$ which has the highest membership degree $\mu\left(a_{i}, C^{h}\right)$.

\footnotetext{
${ }^{1}$ Note that in PROAFTN method [3] each profile $b_{k}^{h}$ is defined, for each criterion $g_{j}$, by an interval $\left[S_{j}^{1}\left(b_{k}^{h}\right), S_{j}^{2}\left(b_{k}^{h}\right)\right]$ which is an exception with regards the other MCC.
}

NCD-based MCC requires parameters such as criteria weights, substitution ratios, indifference, preference and veto thresholds. In MCDA literature, two approaches are proposed to elicit the parameters of MCC: the direct and the indirect elicitation approaches. In the first approach, through an interactive questioning, the decision-maker provides the values of these parameters. The aim of this interaction is to ensure that the parameters values provided by the decision-maker represent properly his/her own judgements and preference system. However, in many other decision-making situations, the determination of the MCC parameters values represents a difficult task due to many reasons such as the size of the problem (i.e. high number of parameters), the imprecise nature of the data, the confusing meaning of the parameters, the analyst ability to perform efficiently the elicitation process, etc. Thus, this direct approach is often time-consuming and consequently it may discourage the decision-maker from participating. To overcome the drawback of the direct approach, the indirect elicitation approach proposes an automatic method to infer the values of these parameters based on examples or prototypes known as training objects (part of a training set). In MCDA literature, this second approach is called preference desegregation approach (e.g. [4], [7], [8]). In artificial intelligence, indirect approach is known as machine learning (e.g. [2]). In this work, the indirect approach will be used to estimate the values of MCC parameters. Note that these values may be obtained by solving the following mathematical model:

$(\Lambda):\left\{\begin{aligned} & \underset{p_{l} \in P}{\text { Minimize }} \Gamma=\sum_{a_{i} \in \mathrm{Z}} \sum_{h=1}^{H}\left(\mu_{i h}\left(p_{1}, p_{2}, \ldots p_{S}\right)-\eta_{i}^{h}\right)^{2} \\ & \text { Subject to } \text { Structural Constraints (SC) } \\ & \text { Decision-Maker's Constraints (DMC) }\end{aligned}\right.$

where $\eta_{i}^{h}=\left\{\begin{array}{ll}1 & \text { if } a_{i} \in C^{h} \\ 0 & \text { otherwise }\end{array}, \mu_{i h} \equiv \mu\left(a_{i}, C^{h}\right)\right.$,

$P=\left\{p_{i}\right\}_{i=1 . . s}$ is the parameters set and $Z$ is the training set (i.e. a set of objects which assignments is known in advance $)^{2} . \Gamma$ is the objective function of cumulative classification errors and should be minimised, i.e. the difference between the estimated membership degree of $a_{i}$ (i.e. $\left.\mu\left(a_{i}, C^{h}\right)\right)$ obtained by applying NCD-based MCC and the true membership degree $\eta_{i}^{h}$ of $a_{i}$ given a priori in the training set $Z$. Two groups of constraints are considered in $(\Lambda)$ : Structural Constraints (SC) and Decision-Maker's Constrains (DMC). In general, the first group of constrains are articulated in function of the

\footnotetext{
${ }^{2}$ The training set is obtained by partitioning the entire database in two subsets: the first one, called training subset, is used to elicit the values of the parameters and the second subset, called test subset, is used to evaluate the performance of the MCC.
} 
characteristics of the parameters and their mutual relationship. For instance, the indifference threshold should be smaller or equal than the preference threshold for each criterion, i.e. $q_{j}^{h} \leq p_{j}^{h}(j=1 . . n)$. The second group of constrains expresses the decision-maker preferences with respect to the NCD-based MCC parameters. For instance, the decision-maker can specify, for a particular category, that $\sum_{j \in J} w_{j}^{h} \geq \sum_{j \in I} w_{j}^{h}$ where $I, J \subset\{1,2, . ., n\}$ and $I \cap J=\emptyset$.

It's noteworthy that [4] and [12] have proposed similar mathematical models to infer the parameters values of their respective NCD-based MCC. When the values of different thresholds are known, Henriet [12] has shown that for specific configurations of global concordance, discordance and similarity indices, the mathematical model $(\Lambda)$ can be easily relaxed into linear program and then solved by classical optimization methods. In addition, Henriet [12] has proposed two methods based respectively on K-Means and Genetic algorithms to identify the profiles which characterize each category. In this same perspective, Belacel et al. [4] have presented a methodology to infer parameters of PROAFTN classifier. In their methodology, these authors have made some simplifications: only concordance concept is considered, the criteria weights are assumed to be equal and each category is characterized by only one profile. Hence, the parameters that are inferred in this learning process are: the upper and the lower bounds of the interval $\left[S_{j}^{1}\left(b_{k}^{h}\right), S_{j}^{2}\left(b_{k}^{h}\right)\right]$ and the two preference thresholds $p_{j}^{-}\left(b_{k}^{h}\right)$ and $p_{j}^{+}\left(b_{k}^{h}\right)$. Belacel et al. [4] solved a mathematical model similar to $(\Lambda)$ by using a training set and the Reduced Variable Neighbourhood Search (RVNS) meta-heuristic recently proposed by [20]. Belacel et al. [4] have reported that the Correct Classification Rate $(\mathrm{CCR})^{3}$ of PROAFTN is in general better than the CCR of other classification methods reported on the same databases.

Since the objective function $\Gamma$ of $(\Lambda)$ is neither convex nor concave and may have many local optima, it will be difficult to find a global optimum for $(\Lambda)$. $(\Lambda)$ is a NPhard problem. Therefore, it's not possible to use classical optimization methods (e.g. gradient algorithms and interior-point algorithms) to solve $(\Lambda)$. To overcome this difficulty, we propose in the next section an ALM based on RCGA to approximate the optimal solution of $(\Lambda)$ and consequently to infer the parameters values of NCD-based MCC.

\footnotetext{
3 This is a performance measure of a classifier. It's defined by the following ratio: the number of objects that are correctly classified divided by the total number of objects.
}

\section{ALM based on RCGA for MCC parameters elicitation}

Genetic Algorithms (GAs) are stochastic algorithms based on the mechanism of the genetic evolution (selection, cross-over and mutation) to solve complex and large optimization problems. GAs were initially introduced by John Holland [14], but they were popularized thanks to the book of Goldberg [9]. The main idea of GAs is to start with an initial population of potential solutions arbitrarily selected. Then, evaluate the relative performance of each solution through a fitness function. Then, on the basis of solutions performances, generate a new population using three evolutionary operators: selection, crossover and mutation. The selection operator identifies both the relatively "good" solutions that will be used to generate the new population and the relatively "bad" solutions that will be removed from the current population. The crossover operator swaps the structures of two "parent solutions" in order to form two similar "offspring solutions" that will be involved in the new population. The mutation operator alters arbitrarily the features of one or more solutions in order to increase the structural variability of the population. The above three operators are repeated until a stopping condition is met. A simplified structure of genetic algorithm is shown in Figure 2.

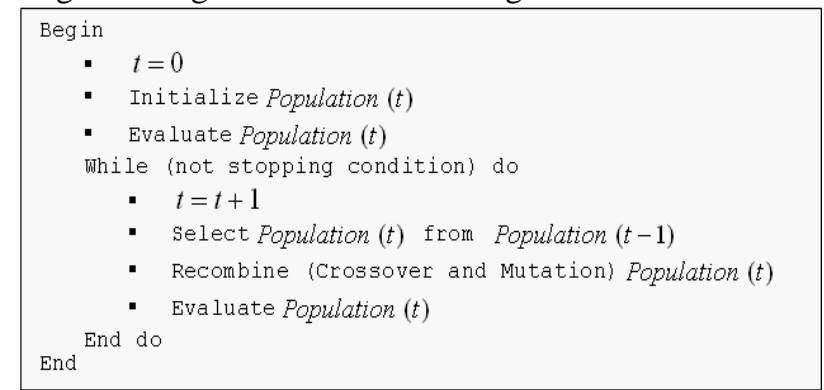

Figure 2: A Simplified GA Structure.

The application fields of GAs are considerable. For instance, these algorithms are used in:

- Optimization, when the functions to optimize are complex, irregular and with high dimensionality.

- Artificial Intelligence (AI), where the adaptive abilities of GAs are exploited.

- Image Recognition (IR), when unknown objects are to be classified to pre-defined categories.

-...

It's important to note that the success of GAs is mainly due to their ability to exploit vast unknown search spaces in order to orient subsequent searches into useful subspaces. This feature makes GAs more efficient and effective search technique to explore large, complex, and poorly understood search spaces, where classical search tolls are inappropriate. Since many years, binary coded solutions (or chromosomes) have dominated GAs research. However, Michalewicz [18] has shown that this kind of representation in case of continuous variables might have at least three inconveniences: 
- It's difficult to use binary coding for optimization problems with high dimension and numerical precision ${ }^{4}$. For this kind of binary coded problems GAs will have weak performance [19].

- The Hamming ${ }^{5}$ distance between two neighbourhood real numbers may be large in binary coding ${ }^{6}$.

- When the crossover and the mutation operators are applied on binary coded continuous chromosomes they may generate new infeasible chromosomes.

The above drawbacks of the binary coded chromosomes have motivated the development of other coding types. One of the most important is the real coding, which is particularly natural when optimization problems involve real variables. GAs with this type of coding are called Real-Coded GAs (RCGAs). In the recent years, RCGAs have been used to solve various continuous optimization problems (e.g. [1], [25]). In RCGAs, each chromosome is treated as a vector of real numbers. Since the conventional crossover and mutation operators for binary coding are not applicable for real coding, many other adapted operators are proposed in the literature for real coded chromosomes (see [13]). In this work, RCGAs will be used to infer the parameters of NCD-Based MCC since all of them are real numbers. To implement the RCGAs, some technical choices have been made on its parameters (e.g. selection methods, crossover and mutation operators, etc.). These choices will be specified in section 5. In next section, we briefly describe the military database that will be used to test the performance of the different classifiers.

\section{Database description}

The military database that will be used in this work includes 2545 Forward Looking Infra-Red (FLIR) images belonging to eight different classes of ships. These images were provided by the U.S. Naval Weapons Center and Ford Aerospace Corporation. Typical silhouettes of the best image of each class and other related information about classes are listed in Table 1.

Based on these FLIR images, Park and Sklansky [21] proposed to extract 11 features $^{7}$ (or attributes/ criteria). These attributes are obtained as follows:

\footnotetext{
${ }^{4}$ For instance, with 100 variables belonging to the interval $[-500,500]$ and a precision of 6 decimal numbers, the size of a binary coded chromosome is 3000 . This is generates a search space of about $10^{1000}$.

${ }^{5}$ The Hamming distance between two binary coded strings is defined as the number of bits which are different in the two strings.

${ }^{6}$ For instance, the Hamming distance between 0111 (which is equal to 7) and 1000 (which equal to 8), is equal to 4.

${ }^{7}$ A feature is an abstraction of the raw data in order to represent the original information.
}

\begin{tabular}{|c|c|c|c|}
\hline Class & Class of ship & $\begin{array}{c}\text { Number of } \\
\text { images }\end{array}$ & Typical silhouette \\
\hline 1 & Destroyer (D) & 340 & \\
\hline 2 & Container (CO) & 455 & \\
\hline 3 & Civilian Freighter (CF) & 186 & \\
\hline 4 & $\begin{array}{c}\text { Auxiliary Oil } \\
\text { Replenishment (AOR) }\end{array}$ & 490 & \\
\hline 5 & $\begin{array}{c}\text { Landing Assault } \\
\text { Tanker (LAT) }\end{array}$ & 348 & \\
\hline 6 & $\begin{array}{c}\text { Frigate (F) } \\
7\end{array}$ & 279 & \\
\hline 8 & $\begin{array}{c}\text { Destroyer with } \\
\text { Cruiser (CR) }\end{array}$ & 239 & \\
\hline
\end{tabular}

Table 1. Data base description.

- The first seven (7) attributes are represented by Hu's [15] moments $m_{i}$. These moments are invariant under scaling (different zoom factors), rotation (different look angles) and translation (silhouette not necessarily centered). The moments $m_{i}$ are computed by using the second and the third order moment formula, let:

$$
\mu_{n m}=\sum_{(x, y) \in S}(x-\bar{x})^{n}(y-\bar{y})^{m}
$$

where $(n+m)$ is the moment order; $x$ (respectively $y)$ is the horizontal (respectively the vertical) coordinates in the silhouette $S ; \bar{x}$ and $\bar{y}$ are the coordinates of the centroid of $S$. For instance, the first four (among the seven attributes) Hu's [15] invariant moments $m_{i}(i=1 . .4)$ are given as follows:

- $\quad g_{1}=m_{1}=\frac{r}{B}$, where $r=\sqrt{\mu_{20}+\mu_{02}}$ the radius of gyration and $B$ is the distance between the camera and the ship.

$$
\begin{aligned}
& -\quad g_{2}=m_{2}=\frac{\left(\mu_{20}-\mu_{02}\right)^{2}+4 \mu_{11}^{2}}{r^{4}} \\
& -\quad g_{3}=m_{3}=\frac{\left(\mu_{30}-3 \mu_{12}\right)^{2}+\left(3 \mu_{21}-\mu_{30}\right)^{2}}{r^{6}} \\
& -\quad g_{4}=m_{4}=\frac{\left(\mu_{30}+\mu_{12}\right)^{2}+\left(\mu_{21}+\mu_{30}\right)^{2}}{r^{6}}
\end{aligned}
$$

The seven Hu's [15] invariant moments are noted by $g_{i}=m_{i} \quad(i=1, \ldots, 7)$. It's worth noting that the weakness of invariant features is that they contain only information that deals with the general shape of the ship and thereby they represent poorly the other details of the observed object. To overcome this disadvantage, $\mathrm{Hu}$ [15] proposed four other attributes which provide more information details about ship; 
- The last four (4) attributes represent the parameters of an Auto Regressive (AR) Model. They were extracted by fitting an AR (Auto-Regressive) model to onedimensional sequence which represents the projection of a ship image onto horizontal axis. Let $r(i)$ $(i=1 . . N)$ denote the sequence of the projected ship image sampled at $N$ equally spaced points. Based on these sequences, an AR model is defined recursively by:

$$
r(i)=\sum_{j=1}^{m} \theta_{j} r(i-j)+\alpha+\sqrt{\beta} \varepsilon(i)
$$

The above model express the projection $r(i)$ $(i=1 . . N)$ as a linear combination of the previous projections $r(i-j)(j=1 . . m)^{8}$, plus a bias $\alpha$ and the error $\varepsilon(i)$ associated with the model. The parameters are estimated by a least square fit of the model to the one-dimensional sequence $r(1), r(2)$, $\ldots, r(N)$. Thus, if $\hat{\theta}, \hat{\alpha}$ and $\hat{\beta}$ denote the least squares estimates of $\theta, \alpha$ and $\beta$ respectively, the four AR parameters $(m=3, N=30)$ are presented as follows:

$$
\begin{aligned}
& -\quad g_{i+7}=\hat{\theta}_{i}, i=1 . .3 \\
& -\quad g_{11}=\frac{\hat{\alpha}}{\sqrt{\hat{\beta}}}
\end{aligned}
$$

Park and Sklansky [21] have shown that all AR parameters are invariant to rotation, translation and scaling, so that they may be used as features for classification purpose.

\section{Computational results}

Only two NCD-based MCC are implemented in this work: PROAFTN classifier [3] and K-PIP classifiers [12]. This choice is justified by the two following facts. The first one is that K-PIP classifier [12] is an enhanced version of the FBI classifier [22] (see [5]). Second, since we want to experiment the effects of both concordance and discordance concepts on classification results, TRINOMFC classifier $^{9}$ ([17]) is removed from our list.

In the ALM proposed in this paper, only thresholds will be estimated for both PROAFTN and K-PIP classifiers:

- For PROAFTN classifier [3], we consider the upper and the lower bounds of the interval $\left[S_{j}^{1}\left(b_{k}^{h}\right), S_{j}^{2}\left(b_{k}^{h}\right)\right]$, the two preference thresholds $p_{j}^{-}\left(b_{k}^{h}\right)$ and $p_{j}^{+}\left(b_{k}^{h}\right)$ and the two veto thresholds $v_{j}^{-}\left(b_{k}^{h}\right)$ and $v_{j}^{+}\left(b_{k}^{h}\right)$ for $j=1 . .11, h=1 . .8$ and $k=1 . . L_{h}$.

- For K-PIP classifier [12], we infer the indifference threshold $q_{j}^{h}$, the preference threshold $p_{j}^{h}$ and finally

\footnotetext{
${ }^{8}$ Here $m$ denotes the number of weight parameters.

${ }^{9}$ TRI-NOMFC classifier uses only concordance concept to assign an object to a predefined category.
}

the two veto threshold $v_{j h}^{-}$and $v_{j h}^{+}$for $j=1, \ldots, 11$ and $h=1, \ldots, 8$.

It is obvious that the dimensionality (or the number of parameters to infer) of the ALM for the above two classifiers is not the same. For instance, in K-PIP classifier there is only 352 parameters $(4 \times 11 \times 8)$ to estimate whereas in PROAFTN classifier there is $\mathbf{5 2 8 0}$ parameters $(6 \times 11 \times 8 \times 10)$ to estimate if we assume that each category is represented by only 10 profiles.

Since the criteria weights are not included in the ALM, they are estimated by the Entropy method [28]. Hence, more the criterion discriminates between images more it will be important. In the other hand, the profiles of each category are identified by using an improve version of K-Means algorithm ${ }^{10}$. The number of profiles in each category is determined by a percentage of the total number of objects in this category. This percentage varies from $1 \%$ to $10 \%$.

Many others technical choices have been made to implement the RCGA on which is based the ALM:

- Four selection methods are implemented: (1) Roulette Wheel Selection (RWS), (2) Stochastic Remainder Without Replacement Selection (SRWRS), (3) Linear Rank based Selection (LRS) and (4) Tournament Selection (TS) ${ }^{11}$;

- Five crossover operators are implemented: (1) Flat Crossover (FC), (2) Arithmetical Crossover (AC), (3) BLX- $\alpha$ Crossover (BLXC), (4) Extended Line Crossover (ELC) and (5) Simple Crossover (SC);

- Four mutation operators are implemented: (1) Random Uniform Mutation (RUM), (2) Non Uniform Mutation (NUM), (3) Mühlenbein Mutation (MM) and (4) Gaussian Mutation (GM);

- The crossover and mutation probabilities vary respectively from 0.6 to 0.8 and from 0.05 to 0.1 ;

- The size of the generated populations vary from 30 to 80 ;

- The maximum iteration number, fixed to 100 , is used as stopping criteria for the RCGA.

- The military database is randomly divided into two subsets: a training subset (which size varies from $50 \%$ to $70 \%$ of the entire database) used to infer the values of the MCC parameters and a test subset (which size varies from $30 \%$ to $50 \%$ of the entire database) used to evaluate the performance of different $\mathrm{MCC}^{12}$. Note that, for each MCC, 20 different random splits are generated to test its performance.

The RCGA and the PROAFTN and K-PIP classifiers are coded in VB (Visual Basic) and tested on a Pentium IV

\footnotetext{
${ }^{10}$ In this improved version, we remove each profile that form an empty group.

${ }^{11}$ To learn more about these evolutionary operators (crossover and mutation) and methods (selection), we refer the reader to the work of Herrera et al. [13]

${ }^{12}$ Note that each subdivision constitutes a partition of the entire database, i.e. the union of the training subset and the test subset form the entire database.
} 
processor with $2.8 \mathrm{GHz}$ and $512 \mathrm{Mb}$ of RAM. The developed software involves some visualization and statistical tools on the entire, training and test databases. For instance, Figure 3 presents some statistical measures on the training database for each class and each attribute (or feature). These measures will be used to limit the variation domains of the estimated parameters et thereby to make easy the generation of the initial populations.

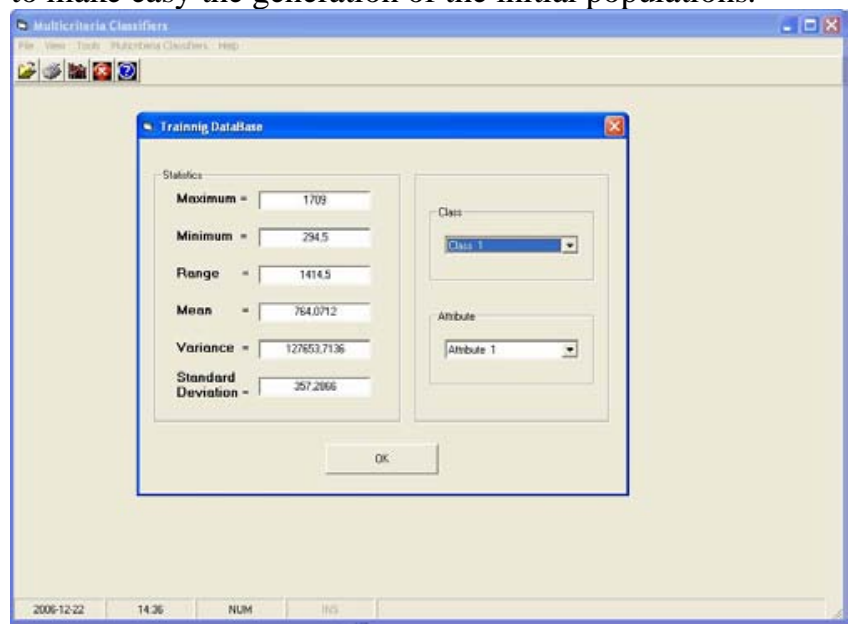

Figure 3. Statistical results.

It's important to underline that two prior woks ([26], [21]) have used the same military database to test the performance of four different classifiers: DempsterShafer-based (DS) classifier, Modified-Bayes-based (MB) classifier, K-Nearest Neighbours (K-NN) classifier and Neural Net (NN) classifier. The results, expressed in Average Identification Rate (AIR), obtained by these classifiers are presented in Table 2.

\begin{tabular}{|c|c|c|c|c|}
\hline Works & $\begin{array}{c}\text { MB } \\
\text { classifier }\end{array}$ & $\begin{array}{c}\text { DS } \\
\text { classifier }\end{array}$ & $\begin{array}{c}\text { K-NN } \\
\text { classifier }\end{array}$ & $\begin{array}{c}\text { NN } \\
\text { classifier }\end{array}$ \\
\hline Valin et al. [26] & $77.7 \%$ & $74.5 \%$ & $94.8 \% 13$ & $92.7 \%$ \\
\hline $\begin{array}{c}\text { Park and } \\
\text { Sklansky [21] }\end{array}$ & $* * *$ & $* * *$ & $88.3 \%{ }^{14}$ & $* * *$ \\
\hline
\end{tabular}

Table 2. The different results of prior works on the military database.

The application of K-PIP and PROAFTN classifiers on the military database provides the results presented respectively in Tables 3 and 4 . An example of screen showing the application of ALM for PROAFTN classifier is presented in Figure 4. By observing Tables 3 and 4, we conclude that both PROAFTN and K-PIP classifiers give, in general, good results: the AIR of FROAFTN is $86.78 \%$ and the AIR of K-PIP is $80.69 \%$. Hence, these two MCC have an AIR better than those of $\mathrm{MB}$ and DS classifiers but worse than those of K-NN and NN classifiers.

\footnotetext{
${ }^{13}$ In this work, the K-NN classifier is applied with $\mathrm{K}=3$ and by using an Euclidian distance weighted by the inverse of the intercategories covariance matrix.

${ }^{14}$ In this work, the K-NN classifier is applied with a simple Euclidian distance. The value of $\mathrm{K}$ that produces the highest AIR is chosen among the values of $K$ between 1 and 17 .
}

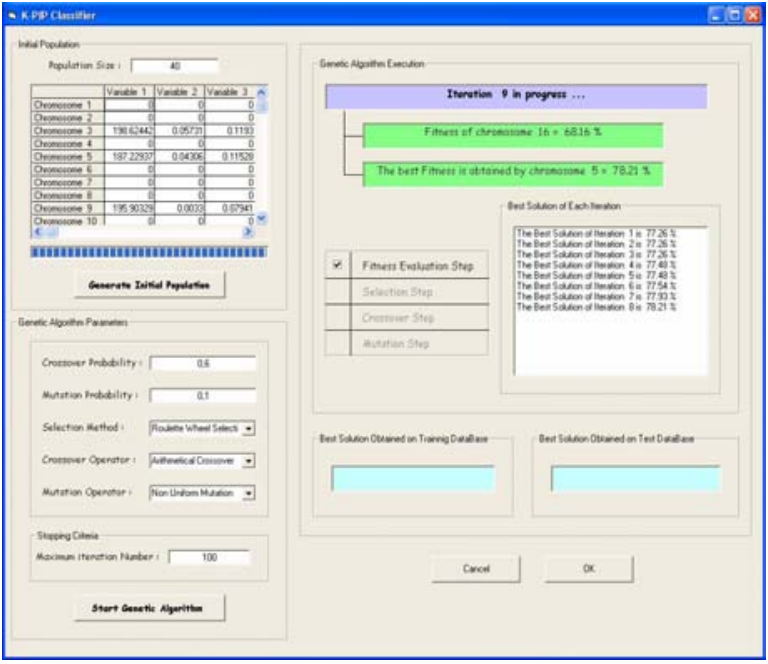

\begin{tabular}{|c|c|c|c|c|c|c|c|c|}
\hline \multirow[b]{2}{*}{ Problems } & \multicolumn{2}{|c|}{ Genetic Algorithm } & \multirow{2}{*}{ Population size } & \multirow{2}{*}{ Selection Method } & \multicolumn{2}{|c|}{ Crossover Step } & \multicolumn{2}{|c|}{ Mutation Step } \\
\hline & Trâning Dattabse & Test database & & & Cross. Oper & Cross: Prob & \begin{tabular}{|l|} 
Muta Oper \\
\end{tabular} & Muta. Prob \\
\hline Problem 1 & $92,62 \%$ & $86,23 \%$ & 30 & RWS & FC & 0,60 & RUM & 0,05 \\
\hline Problem 2 & $91,78 \%$ & $85,03 \%$ & 30 & SRWRS & $A C$ & 0,65 & NUM & 0,07 \\
\hline Problem 3 & $94,33 \%$ & $88,54 \%$ & 30 & LRS & sc & 0,70 & MM & 0,09 \\
\hline Problem 4 & $90,44 \%$ & $85,09 \%$ & 40 & TS & BLX & 0,75 & GM & 0,10 \\
\hline Problem 5 & $95,22 \%$ & $89,67 \%$ & 40 & RWS & ELC & 0,80 & RUM & 0,05 \\
\hline $\begin{array}{l}\text { Problem } 6 \\
\end{array}$ & $93,02 \%$ & $84,88 \%$ & 40 & SRWRS & FC & 0,60 & NUM & 0,07 \\
\hline Problem 7 & $96,17 \%$ & $88,98 \%$ & 50 & LRS & $\mathrm{AC}$ & 0,65 & MM & 0,09 \\
\hline Problem 8 & $94,01 \%$ & $86,11 \%$ & 50 & is & sc & 0,70 & GM & 0,10 \\
\hline Problem 9 & $93,28 \%$ & $85,96 \%$ & 50 & RWS & BLX & 0,75 & RUM & 0,05 \\
\hline Problem 10 & $93,72 \%$ & $86,31 \%$ & 60 & SRWRS & ELC & 0,80 & NUM & 0,07 \\
\hline Problem 11 & $95,12 \%$ & $88,17 \%$ & 60 & LRS & FC & 0,60 & MM & 0,09 \\
\hline Problem 12 & $91,73 \%$ & $84,39 \%$ & 60 & is & AC & 0,65 & GM & 0,10 \\
\hline Problem 13 & $95,09 \%$ & $87,81 \%$ & 70 & RWS & sc & 0,70 & RUM & 0,05 \\
\hline Problem 14 & $96,00 \%$ & $89,42 \%$ & 70 & SRWRS & BLX & 0,75 & NUM & 0,07 \\
\hline Problem 15 & $93,08 \%$ & $86,12 \%$ & 70 & LRS & ELC & 0,80 & MM & 0,09 \\
\hline Problem 16 & $94,65 \%$ & $88,23 \%$ & 70 & is & $\mathrm{FC}$ & 0,60 & GM & 0,10 \\
\hline Problem 17 & $95,00 \%$ & $87,93 \%$ & 80 & RWS & $\mathrm{AC}$ & 0,65 & RUM & 0,05 \\
\hline Problem 18 & $93,75 \%$ & $85,59 \%$ & 80 & SRWRS & sc & 0,70 & NUM & 0,07 \\
\hline Problem 19 & $92,82 \%$ & $85,07 \%$ & 80 & LRS & BLX & 0,75 & MM & 0,09 \\
\hline Problem 20 & $94,68 \%$ & $86,12 \%$ & 80 & is & ELC & 0,80 & GM & 0,10 \\
\hline Mean & $93,83 \%$ & $86,78 \%$ & & & & & & \\
\hline Standad deviation & $1,50 \%$ & $1,65 \%$ & & & & & & \\
\hline Mean range & 7,04 & & & & & & & \\
\hline
\end{tabular}

Table 3. PROAFTN results.

Some other comments may be made on the classification results of both PROAFTN and K-PIP classifiers:

- The AIRs of both classifiers on the training and test databases are stable since they are situated around their average (small standard deviation for both classifiers and databases);

- The AIRs obtained on the training and the test databases are too different for PROAFTN classifier (average range $7.04 \%$ ), while these AIRs are nearly the same for K-PIP classifier (average range 2.53\%). We believe that this is due both to the high number of parameters in PROAFTN classifier and to the overspecification problem. This problem occurs when the parameters of the classifier became much specific to the data set from which they are assessed. Hence, when these parameters are used to classify another data set, the classification results obtained on this latter will be much different from those obtained on the first data set. Since PROAFTN classifier use more parameters than K-PIP classifier, the overspecification problem will be more apparent with the former classifier; 
- According to our computational experiments, all selection methods and evolutionary operator (mutation and crossover) seems to perform equally. However, we can observe that, in general, the AIRs of both MCCs increase when the population size increases;

- Some AIRs obtained by PROAFTN classifier for the training database exceed the best AIR obtained by all other classifiers (i.e. 94.8\%). This is shows the ability of this classifier to provide better results and thereby we believe that it constitutes a promising classifier which merits to be improved (see section 6 for eventual improvements);

- The AIR obtained by PROAFTN classifier is better than that of K-PIP classifier since, for a specific criterion, the first consider that the profiles of the same category don't have necessarily the same thresholds, while the second assumes that the profiles of the same category have identical thresholds. Hence, for a particular category and criterion, PROAFTN classifier provides more thresholds to each profile (i.e. more degree of freedom) than K-PIP classifier.

\begin{tabular}{|c|c|c|c|c|c|c|c|c|}
\hline \multirow[b]{2}{*}{ Problems } & \multicolumn{2}{|c|}{ Genetic Algorithm } & \multirow{2}{*}{ Population size } & \multirow{2}{*}{ Selection Method } & \multicolumn{2}{|c|}{ Crossover Step } & \multicolumn{2}{|c|}{ Mutation Step } \\
\hline & Training Database & Test database & & & Cross. oper & Cross. Prob & \begin{tabular}{|l|} 
Muta. Oper \\
\end{tabular} & Muta. Prob \\
\hline Problem 1 & $83,38 \%$ & $80,63 \%$ & 30 & RWS & $\mathrm{FC}$ & 0,60 & RUM & 0,05 \\
\hline Problem 2 & $81,47 \%$ & $79,84 \%$ & 30 & SRWRS & AC & 0,65 & NUM & 0,07 \\
\hline Problem 3 & $82,31 \%$ & $80,89 \%$ & 30 & LRS & $5 \mathrm{sc}$ & 0,70 & MM & 0,09 \\
\hline Problem 4 & $82,54 \%$ & $79,58 \%$ & 40 & is & BLX & 0,75 & GM & 0,10 \\
\hline Problem 5 & $84,31 \%$ & $81,03 \%$ & 40 & RWS & ELC & 0,80 & RUM & 0,05 \\
\hline Problem 6 & $83,25 \%$ & $80,22 \%$ & 40 & SRWRS & $\mathrm{FC}$ & 0,60 & NUM & 0,07 \\
\hline Problem 7 & $85,02 \%$ & $82,41 \%$ & 50 & LRS & AC & 0,65 & MM & 0,09 \\
\hline Problem 8 & $84,59 \%$ & $81,19 \%$ & 50 & is & $\mathrm{sc}$ & 0,70 & GM & 0,10 \\
\hline Problem, & $83,69 \%$ & $80,07 \%$ & 50 & RWS & BLX & 0,75 & RUM & 0,05 \\
\hline Problem 10 & $82,58 \%$ & $80,97 \%$ & 60 & SRWRS & ELC & 0,80 & NUM & 0,07 \\
\hline Problem 11 & $85,17 \%$ & $83,72 \%$ & 60 & LRS & $\mathrm{FE}$ & 0,60 & $\mathrm{MIM}$ & 0,09 \\
\hline Problem 12 & $80,77 \%$ & $78,61 \%$ & 60 & is & AC & 0,65 & GiM & 0,10 \\
\hline Problem 13 & $85,04 \%$ & $82,51 \%$ & 70 & RWS & sc & 0,70 & RUM & 0,05 \\
\hline Problem 14 & $82,54 \%$ & $79,91 \%$ & 70 & SRWRS & BLX & 0,75 & NUM & 0,07 \\
\hline Problem 15 & $84,00 \%$ & $81,25 \%$ & 70 & LRS & ELC & 0,80 & MM & 0,09 \\
\hline Problem 16 & $80,87 \%$ & $77,88 \%$ & 70 & Ts & $\mathrm{FC}$ & 0,60 & GM & 0,10 \\
\hline Problem 17 & $83,36 \%$ & $80,11 \%$ & 80 & RWS & $\mathrm{AC}$ & 0,65 & RUM & 0,05 \\
\hline Problem 18 & $82,43 \%$ & $80,81 \%$ & 80 & SRWRS & sc & 0,70 & NUM & 0,07 \\
\hline Problem 19 & $85,65 \%$ & $83,09 \%$ & 80 & LRS & BLX & 0,75 & MM & 0,09 \\
\hline Problem 20 & $81,47 \%$ & $79,17 \%$ & 80 & TS & ELC & 0,80 & GM & 0,10 \\
\hline mean & $83,22 \%$ & $80,69 \%$ & & & & & & \\
\hline Standad deviation & $1,46 \%$ & $1,45 \%$ & & & & & & \\
\hline Mean ronge & & & & & & & & \\
\hline
\end{tabular}

\section{Discussions and conclusions}

In this work, we proposed an Automatic Learning Approach (ALM) based on Real Coded Genetic Algorithm (RCGA) to infer the parameters of some NCD-based MCC. The proposed ALM overcomes some simplifications made in prior works (e.g. [4]): both concordance and discordance concepts are taken into account, the criteria weights are used in the computation of the membership degree of an object to a predefined category and finally each category may be characterized by many profiles.

A database of 2545 Forward Looking Infra-Red (FLIR) images representing eight different classes of ships is used to test the performance of two NCD-based MCCs (PROAFTN [3] and K-PIP [12] with respect four other classifiers (Dempster-Shafer (DS), Modified-Bayes
(MB), K-Nearest Neighbours (K-NN) and Neural Net $(\mathrm{NN})$ ). The computational results show that NCD-based MCCs provide AIRs better than those provided by MB and DS classifiers but worse than those obtained by K$\mathrm{NN}$ and NN classifiers. Although NCD-based MCCs don't provide the best AIRs in this application, we believe that they are promising classifiers and merit to be further explored. Note that NCD-based MCCs are not optimised for this database. In fact, if we would like to introduce qualitative information and human judgement, we are confident that NCD-based MCCs will outrank KNN and NN classifiers. NCD-based MCCs are not a black box and all the results are automatically explained. Many improvements could be made to enhance the AIRs of NCD-based MCCs like:

- Integrating the profiles and the criteria weights in the learning process;

- Using other improved version of K-Means algorithm for the profile identification (e.g. Y-Means [10] or JMeans [11]);

- Combining the aggregation operators of the different NCD-based MCCs;

- Using the concept of specified classifier, i.e. for the classification purpose we only use a subset of criteria that discriminate more between objects;

- Implementing a parallel version of NCD-based MCCs to reduce the computation time. For instance, concordance and discordance indices may be computed simultaneously;

- Since GAs do not exploit local information of points in the population set, it will be benefit to integrate a local search algorithm (e.g. Tabu search, simulated annealing...) in each iteration of the GA.

In this paper, we have argued for cross fertilization of multi-criteria decision analysis and information fusion concepts. Multiple criteria classifiers have the strength of being designed to consider human in the loop and to support human decision making.

\section{Reference}

[1] Ali, M.M., Törn, A., (2004), Population set based global optimization algorithms: Some modifications and numerical studies, Computers and Operations Research Vol. 31 (10), pp. 1703-1725.

[2] Arhcer N.P. and Wang S. (1993), Application of the back propagation neural networks algorithm with monotonicity constraints for two group classification problems. Decision Sciences, Vol. 24, pp. 60-75.

[3] Belacel N. (2000), Multi-criteria assignment method PROAFTN: Methodology and medical application, European Journal of Operational Research, Vol. 125, pp. 175-183.

[4] Belacel N., Raval H. B. and Punnen, A. P. (2007), Learning multi-criteria fuzzy classification method PROAFTN from data, Computer and Operations Research, Vol. 34(7), pp. 1885-1898. 
[5] Benabbou, L. Guitouni, A. and Lang, P. (2004), Méthode de classification : revue de littérature, essai de caractérisation et de comparaison, Document de travail 2004-023, FSA, Université Laval, Québec, Canada.

[6] Bouyssou D., T. Marchant, M. Pirlot, P. Perny, A. Tsoukias, P. Vincke (2000). Evaluation and Decision Models. A Critical Perspective. Kluwer Academic Publishers, Boston/London/Dordrecht.

[7] Dias L. C. and Mousseau V., (2006), Inferring Electre's veto-related parameters from outranking examples. European Journal of Operational Research, Vol. 170(1), pp. 172-191.

[8] Dias L., Mousseau V., Figueira J., and Climaco J., (2002), An aggregation/disaggregation approach to obtain robust conclusions with ELECTRE TRI, European Journal of Operational Research, Vol. 138(2), pp. 332-348.

[9] Goldberg, D. (1989), Genetic Algorithm In Search, Optimization And Machine Learning, Addison-Wesley.

[10] Guan, Y., Ghorbani, A. and Belacel, N (2003), YMeans: A Clustering Method for Intrusion Detection, The Canadian Conference on Electrical and Computer Engineering, Montréal, Québec, Canada. May 4-7, 2003.

[11] Hansen, P. and N. Mladenovic (2001), J-MEANS: a new local search heuristic for minimum sum of squares clustering, Pattern Recognition Journal, Vol. 34, 405413.

[12] Henriet, L. (2000), Systèmes d'évaluation et de classification multicritère pour l'aide à la décision, Thèses de Doctorat, Université Paris Dauphine, France.

[13] Herrera F., Lozano M. and Verdegay J.L. (1998), Tackling real-coded genetic algorithms: operators and tools for behavioural analysis, Artificial Intelligence Review, Vol. 12(4), pp. 265-319.

[14] Holland, J. H. (1975), Adaptation In Natural And Artificial Systems, University of Michigan Press.

[15] Hu M.K. (1962), Visual pattern recognition by moment invariant, IEEE Transactions on Information Theory IT-8, pp. 179-187.

[16] Jabeur, K. and Guitouni, A. (2007), A generalized framework for concordance/discordance-based multicriteria classification methods, Fusion 2007, Quebec City.

[17] Léger J. and Martel J-M (2002), A Multi-criteria assignment procedure for a nominal sorting problematic, European Journal of Operational Research, Vol. 138, pp. 349-364.

[18] Michalewicz, Z. (1992), Genetic Algorithms + Data Structures $=$ Evolution Programs, Springer-Verlag .

[19] Michalewicz, Z., Logan, T. \& Swaminathan, S. (1994), Evolutionary operators for continuous convex parameters spaces, in A. Sebald \& L. Fogel, eds., Proceedings of the $3^{\text {rd }}$ Annual Conference on Evolutionary Programming, World Scientific Publishing, pp. 84-107.

[20] Mladenovic N, Hansen P. (2001), Variable neighbourhood search: principles and applications, European Journal of Operational Research, Vol. 130, pp. 449-467.

[21] Park Y., Sklansky J., (1990), Automated design of linear tree classifiers, Pattern Recognition, Vol. 23 (12) pp. 1393-1412.

[22] Perny, P. (1998), Multi-criteria filtering methods based on concordance and non-discordance principles, Annals of Operations Research, Vol. 80, pp. 137-165.

[23] Roy, B. (1968), Classement et choix en présence de points de vue multiples (la méthode ELECTRE), R.I.R.O. Recherche Opérationnelle, Vol. 2, pp. 57-75.

[24] Roy, B. (1974), Critères multiples et modélisation des préférences : l'apport des relations de surclassement, Revue d'Économie Politique, Vol. 1.

[25] Roy, S., Bhunia, A.K. and Mukhopadhyay, S. (2005), A genetic algorithmic approach on a deterministic inventory model for deteriorating items with shortages, INFOR, Vol. 43(3), pp. 271-282.

[26] Valin P., Rhéaume F., Tremblay C., Grenier D., Jousselme A-L and Bossé E. (2006), Comparative implementation of two fusion schemes for multiple complementary FLIR imagery classifiers, Information fusion Journal, Vol. 7(2), pp. 197-206.

[27] Vincke, P. (1989), L'aide multicritère à la décision, Éditions de l'Université de Bruxelles, Belgique.

[28] Zeleny, M. (1982), Multiple Criteria Decision Making, McGraw-Hill. 